Proof. Theorem 3.6 and Example 3.3.

\title{
REFERENCES
}

1. C. Berge, The theory of graphs and its applications, Wiley, New York, 1962.

2. M. Maschler and B. Peleg, A characterization, existence proof and dimension bounds for the kernel of a game, Pacific J. Math. 18 (1966), 289-328.

The Hebrew University of Jerusalem

\section{IMBEDDING CLOSED RIEMANN SURFACES IN $C^{n}$}

\author{
K. V. RAJESWARA RAO ${ }^{1}$
}

I. Introduction. Let $R$ be a closed Riemann surface of genus $g$, $G$ a nonempty, open subset of $R$, and $A$ the set of all complex valued functions that are continuous on $R$ and holomorphic on $G$. With the usual pointwise operations $A$ is an algebra over the complex field. We consider the problem: how many functions in $A$ suffice to separate points of $R$ ?

Let $f$ be a nonconstant member of $A$. If the genus $g=0$, Wermer [4] showed that there exist $f_{1}$ and $f_{2}$ in $A$ which, together with $f$ separate points of $R$; if $g=1$, Arens [2] established the existence of $f_{1}, f_{2}$ and $f_{3}$ in $A$ which, together with $f$ separate points of $R$. In this note we shall present a modification of the Wermer-Arens argument to prove the following

TheOREM. Let the genus $g$ be arbitrary. If A contains nonconstant functions, then there exist four functions in $A$ which separate points of $R$ and which have no common branch points in $G$.

The author is indebted to a referee for pointing out an error in an earlier version of the paper.

II. Two lemmas. Let $\phi$ be a nonconstant member of order $n$ in the field $K$ of meromorphic functions on $R$. Let $w$ be a point of the extended plane which has $n$ distinct inverse images under $\phi$. Denote by $E(\phi, w)$ the finite set which is the union of $\phi^{-1}(w)$ and $\phi^{-1}(\phi(b))$ as $b$ ranges over all the branch points of $\phi$. For (fixed) $\phi$ and $\psi$ in $K$, let $S$

Received by the editors December 7, 1966.

${ }^{1}$ Supported by the Air Force Office of Scientific Research and the Purdue Research Foundation. 
be the set of all $z$, in $R$, for which there exists a $z^{\prime}$ in $R$ with $z \neq z^{\prime}$, but $\phi(z)=\phi\left(z^{\prime}\right)$ and $\psi(z)=\psi\left(z^{\prime}\right)$. We then have

\section{Lemma 1. Let $\psi$ separate points of $E(\phi, w)$; then $S$ is finite.}

Proof. There is known (Ahlfors-Sario [1, p. 322]) to exist a polynomial $F_{v}(u) \equiv F(u, v)$ of degree $n$ in $u$, with rational functions of $v$ as coefficients, which is irreducible in $u$ and is such that $F(\psi(p), \phi(p))$ $=0, p \in R$. Let $z \in S$, so that $\phi^{-1}(\phi(z))$ consists of $n$ distinct points, say $z_{1}=z, z_{2}=z^{\prime}, z_{3} \cdots z_{n}$. The way the polynomial $F$ is constructed (Ahlfors-Sario [1, p. 322]), $u=\psi\left(z_{1}\right), \psi\left(z_{2}\right), \cdots, \psi\left(z_{n}\right)$ constitute the roots of the equation $F(u, \phi(z))=0$. Thus, if $z \in S$, this polynomial in $u$ has a repeated root so that its discriminant must be zero. But this discriminant is a rational function $D(v)$ of $v=\phi(z)$ and, since $F(u, v)$ is irreducible in $u$, it is not identically zero. Hence the set of $\phi(z)(z \in S)$ for which $D(\phi(z))=0$ is finite; since $\phi$ has finite valence $n$, this implies that $S$ is finite.

For any $z$ in $R$, let $K_{z}$ be the set of all those functions in $K$ that are holomorphic on $R \backslash\{z\}$.

Lemma 2. If $E$ is a finite subset of $R \backslash\{z\}$, then there exists a member of $K_{z}$ which separates points of $E$ and which has no branch points in $E$.

Proof. For distinct points $z_{1}, z_{2}$ in $R \backslash\{z\}$ and an integer $n>2 g$, consider the divisors

$$
D_{1}=z_{1}-n z \text { and } D_{2}=z_{1}+z_{2}-n z .
$$

By the Riemann-Roch theorem (Ahlfors-Sario [1, p. 329]) the dimension, $\operatorname{dim} D_{1}$, of the complex vector space of those members of $K$ that are multiples of $D_{1}$ satisfies

$$
\operatorname{dim} D_{1} \geqq-\operatorname{deg} D_{1}-g+1=n-g .
$$

Since deg $D_{2}<2-2 g$, we have, again by the Riemann-Roch theorem, (Ahlfors-Sario [1, p. 329])

$$
\operatorname{dim} D_{2}=-\operatorname{deg} D_{2}-g+1=n-g-1 .
$$

This and the preceding inequality show that there exists a function belonging to $K_{z}$ which vanishes at $z_{1}$ but not at $z_{2}$. Since $K_{z}$ is an algebra (under the usual operations) over the complex numbers, it follows that there exists $\phi$ in $K_{z}$ which separates points of $E$.

Let $z_{1}, z_{2}, \cdots, z_{k}$ be those points of $E$ at which the multiplicity of $\phi>1$, and $z_{k+1}, \cdots, z_{m}$ be the other points of $E$. Considering, for $n>2 m+2 g-2$ and $j=1, \cdots, k$, the divisors $-n z-z_{j}+\sum_{i=1}^{m} 2 z_{i}$ and $-n z+\sum_{i=1}^{m} 2 z_{i}$ and applying the Riemann-Roch theorem as before, 
one obtains a function $\phi_{j}$ in $K_{z}$ which has a simple zero at $z_{j}$ and multiple zeros at $z_{i}, i \neq j, i=1, \cdots, m$. Then $\phi+\sum_{j=1}^{k} \phi_{j}$ serves as a function whose existence is claimed in the lemma.

III. Proof of the theorem. Let $p_{1} \in G$ and $\phi_{1}$ be a member of $K$ which has its sole pole, of order $n_{1}$ say, at $p_{1}$. Then, by two applications of Lemmas 1 and 2, choose $\phi_{2}$ and $\phi_{3}$ in $K$ such that $\phi_{i}$ has its sole pole, of order $n_{i}$ say, at $p_{i} \in G, i=2,3, p_{i} \neq p_{j}$, for $i \neq j, i, j=1,2,3$, such that any two of $\phi_{1}, \phi_{2}$ and $\phi_{3}$ separate all but a finite number of pairs $\left(z, z^{\prime}\right) \in R \times R\left(z \neq z^{\prime}\right)$ and such that no two of them have a common branch point. Let $E_{i j}$ be the set of all $z$ in $R$ such that there exists $z^{\prime} \neq z$ with $\phi_{i}(z)=\phi_{i}\left(z^{\prime}\right)$ and $\phi_{j}(z)=\phi_{j}\left(z^{\prime}\right)$. Put $E \equiv\left\{p_{1}, p_{2}, p_{3}\right\} \cup_{i<j} E_{i j}$, so that $E$ is a finite subset of $R$. Suppose that $A$ contains nonconstants functions. Then $A$ separates points of $R$ (Arens [2, p. 255]) and, since $A$ is an algebra, there exists $f_{0}$ in $A$ which separates points of $E$. Set, now, for an integer $n>\max \left(n_{1}, n_{2}, n_{3}\right)$,

$$
f_{i}=\left[f_{0}-f_{0}\left(p_{i}\right)\right]^{n} \cdot \phi_{i}, \quad i=1,2,3 .
$$

It is readily seen that $f_{1}, f_{2}, f_{3} \in A$. To see that $f_{0}, f_{1}, f_{2}$, and $f_{3}$ separate points of $R$, observe that, if $f_{i}\left(z_{1}\right)=f_{i}\left(z_{2}\right), i=0,1,2,3$, then $f_{0}\left(p_{j}\right)$ $\neq f_{0}\left(z_{1}\right)$, for at least two values of the index $j$, say, for $j=1,2$. Then, we obtain that $\phi_{j}\left(z_{1}\right)=\phi_{j}\left(z_{2}\right), j=1,2$, so that either $z_{1}=z_{2}$ or $z_{1}, z_{2} \in E_{12}$; since $f_{0}$ separates points of $E_{12}$ we must indeed have $z_{1}=z_{2}$.

Suppose, if possible, that for some $z \in G$, the differential $d f_{i}(z)$ is zero for $i=0,1,2,3$. Again, we have for two values of $j$, say, for $j=1,2, f_{0}(z) \neq f_{0}\left(p_{j}\right)$, and then we have $d \phi_{j}(z)=0, j=1,2$, which is a contradiction to the choice of the $\phi_{j}$. This completes the proof of the theorem.

REMARK. If $G$ is not dense in $R$, then results of Narasimhan [3] readily imply that three functions in $A$ separate points of $R$. It is an open problem to determine the minimal number of functions in $A$ that separate points of $R$.

\section{REFERENCES}

1. L. V. Ahlfors and L. Sario, Riemann surfaces, Princeton Univ. Press, Princeton, N.J., 1960.

2. R. Arens, The closed maximal ideals of algebras of functions holomorphic on a Riemann surface, Rend. Circ. Mat. Palermo (2) 7 (1958), 245-260.

3. R. Narasimhan, Imbedding of open Riemann surfaces, Nachr. Akad. Wiss. Göttingen Math. Phys. Kl. II (1960), 159-165.

4. J. Wermer, Polynomial approximation on an arc in $C^{3}$, Ann. of Math. (2) 62 (1955), 269-270.

Purdue University 